\title{
BMJ Open Is frequent attendance of longer duration related to less transient episodes of care? A retrospective analysis of transient and chronic episodes of care
}

\author{
Frans T Smits, ${ }^{1}$ Henk J Brouwer, ${ }^{1}$ Aart H Schene, ${ }^{2}$ Henk C P M van Weert, ${ }^{1}$ \\ Gerben ter Riet ${ }^{1}$
}

To cite: Smits FT, Brouwer HJ, Schene AH, et al. Is frequent attendance of longer duration related to less transient episodes of care? A retrospective analysis of transient and chronic episodes of care. BMJ Open 2016;6: 012563.

doi:10.1136/bmjopen-2016012563

- Prepublication history and additional material is available. To view please visit the journal (http://dx.doi.org/ 10.1136/bmjopen-2016012563).

Received 20 May 2016 Revised 31 August 2016 Accepted 4 October 2016

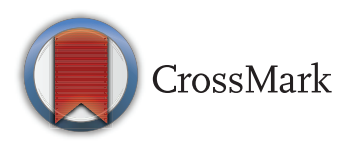

${ }^{1}$ Department of General Practice, Academic Medical Center, University of Amsterdam, Amsterdam, The Netherlands

${ }^{2}$ Department of Psychiatry, Radboud University Medical Center, Nijmegen,

The Netherlands

Correspondence to

Frans T Smits;

f.t.smits@amc.uva.nl

\section{ABSTRACT}

Objectives: Frequent attenders (FAs) suffer more and consult general practitioners (GPs) more often for chronic physical and psychiatric illnesses, social difficulties and distress than non-FAs. However, it is unclear to what extent FAs present transient episodes of care (TECs) compared with non-FAs.

Design: Retrospective analysis of all episodes of care (ECs) in 15116 consultations in 1 year. Reasons for encounter (RFEs) linked to patients' problem lists were defined as chronic ECs (CECs), other episodes as TECs.

Setting: 1 Dutch urban primary healthcare centre served by 5 GPs.

Participants: All 5712 adult patients were enlisted between 2007 and 2009. FAs were patients whose attendance rate ranked within the top decile of their sex and age group in at least one of the years between 2007 and 2009.

Outcome measures: Number of RFEs linked to TECs/CECs for non-FAs and 1-year (1yFAs), 2-year (2yFAs) and 3-year FAs (3yFAs), and the adjusted effect of frequent attendance of different duration on the number of TECs.

Results: The average number of RFEs linked to TECs (non-FAs 1.4; 3yFAs 7.3) and to CECs (non-FAs 0.9; 3yFAs 6.2) increased substantially with the duration of frequent attendance. The ratio of TECs to all ECs differed little for FAs (52-54\%) and non-FAs (64\%). Compared with non-FAs, the adjusted additional number of TECs was 3.4 (95\% $\mathrm{Cl} 3.2$ to $3.7,1 \mathrm{yFAs})$, $6.6(95 \% \mathrm{Cl} 6.1$ to $7.0,2 \mathrm{yFAs})$ and $9.4(95 \% \mathrm{Cl} 8.8$ to 10.1, 3yFAs).

Conclusions: FAs present more TECs and CECs with longer duration of frequent attendance. The constant ratio of TECs might be a sign of a low threshold for FAs to consult their GP. The large numbers of TECs in FAs might be associated with their high level of anxiety and low mastery. The consultation pattern of FAs may best be characterised by describing both TECs and CECs.

\section{Strengths and limitations of this study}

- This is the first study which analyses both transient (TECs) and chronic episodes of care (CECs) in frequent attenders (FAs) of increasing duration.

- By using an automated coding system, coding differences between general practitioners (GPs) are expected to be small in chronic episodes of care in particular.

- Since the involved GPs agreed to register and code CECs only, under-registration of TECs and underestimation of the number of TECs in FAs may be likely.

- The study did not include data on anxiety or mastery.

\section{BACKGROUND}

General practitioners (GP) spend about $40 \%$ of all consultations on the $10 \%$ most frequently attending patients. These frequent attenders (FAs) suffer from more chronic physical and psychiatric illnesses, social difficulties and emotional distress than non-FAs. ${ }^{1-3}$ The sex-corrected and agecorrected prevalences of chronic medical problems of persistent FAs (FA during at least three consecutive years) are up to 3-5 times higher than in non-FAs. ${ }^{1} 45$ Panic disorder, anxiety, negative life events, illness behaviour and lack of mastery are independently associated with persistence of frequent attendance. ${ }^{6}$ However, little is known about the content of consultations of these FAs and the way GPs describe their illness behaviour.

In our previous work, we characterised a patient's morbidity using the entries from a list of all ongoing chronic or repetitive medical problems, the problem list. This list, 
by definition, does not represent all reasons for encounter (RFEs) during a consultation or episodes of care (ECs). In every consultation, a patient can present several RFEs linked to ECs, either chronic or transient ones (see figure 1). Patients presenting transient ECs (TECs) demonstrate more illness worry, unmet expectations and dissatisfaction with the consultation compared with patients receiving a diagnosis. ${ }^{7}$ Therefore, presenting many RFEs linked to TECs can be explained by a general feeling of discomfort or anxiety which the GP has not yet recognised and registered as a chronic psychosocial problem. However, as far as we are aware, the consultation patterns and the ratio of TECs to all ECs in particular have never been quantified, nor its association with duration of FA-ship. We hypothesised that, especially patients who return frequently over a long period of time, predominantly visit their GPs for chronic problems.

To investigate the consultation pattern of the different groups of FAs, we calculated during 1 year the number of RFEs presented in face-to-face consultations with the GP in an urban primary healthcare centre and determined whether they were linked more to chronic ECs (CECs) or TECs.

\section{METHODS}

\section{Design}

Retrospective study using clinical data of all consultations of adult patients of an urban primary healthcare centre served by five GPs, in the Netherlands in 2009.

\section{Patient population and setting}

We used the data of 5712 patients at least 18 years of age, enlisted in 2007-2009 in five GP practices in one primary healthcare centre in Amsterdam, the Netherlands. These practices participated in the GP-based continuous morbidity registration network of the Department of General Practice at the Academic Medical Center of the
University of Amsterdam. In this GP network, electronic medical record data are extracted for research purposes. The studied patients had a lower socioeconomic level, were of more non-Western descent and were slightly younger than the average Dutch population.

\section{Selection of 1-year, 2-year and 3-year FAs}

We used only face-to-face consultations with the GP (consultations in the surgery and house calls). Patients under the age of 18 years were excluded, because their consultations often involved the parents as well as the patient.

Since only a proportional threshold definition selects the exceptional users within each sex and age group and allows more meaningful comparisons between practices, periods and countries, we defined FAs as the age-adjusted and sex-adjusted (18-30; 31-45; 46-60; 61 years +$)$ top 10 centile attenders within a time frame of 1 year (1-year FAs, 1yFAs). ${ }^{38}$

FAs were determined for each of the years 2007, 2008 and 2009. One-year FAs attended frequently in one of those years. We defined 1yFAs, 2-year (2yFAs) and 3-year FAs (3yFAs) as those patients who were FAs in 1, 2 or 3 of those years, respectively. All other enlisted patients were non-FAs.

\section{Morbidity}

In January 2010, we extracted from the electronic medical records the text of all registered encounters (face-to-face consultations, house calls, telephone and mail contacts) with the GP and staff in 2009. As a patient can present multiple RFEs per consultation, the number of RFEs may exceed the number of consultations. After the diagnostic interpretation by the GP, these RFEs represent an EC. The participating GPs use automated computer-assisted algorithms, which present one or more codes for each diagnostic interpretation of

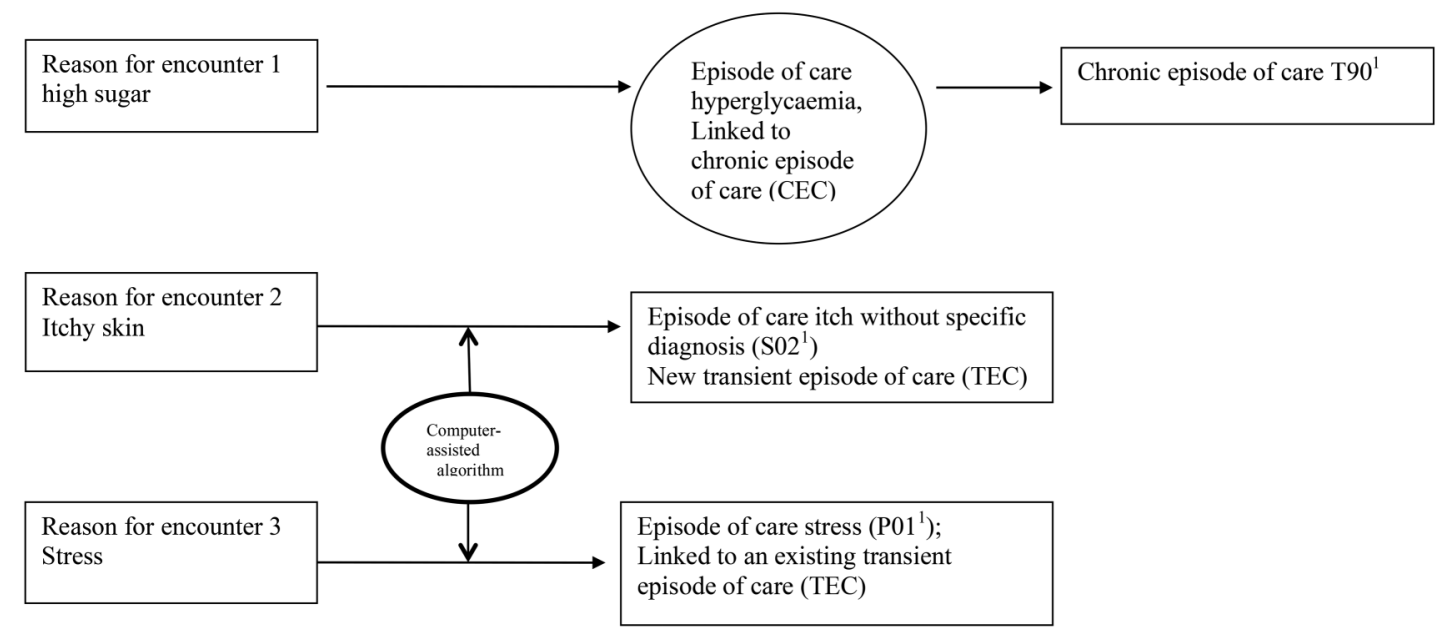

${ }^{1}$ Code according the International Classification of Primary Care

Figure 1 Example of a consultation with three reasons for encounter of which one is linked to a chronic episode of care, one is linked to an existing transient episode of care and one is a new transient episode of care. 
which one has to be selected. The software also suggests either to label specific preselected ECs as a CEC or to link the EC to an existing EC. In 2009, the involved GPs agreed to register and code at least all CECs. If the GP had not documented a code, according to the International Classification of Primary Care (ICPC), an experienced GP and researcher (FTS) formulated one after reading the full text of the consultation and using the same computer-assisted algorithm. ${ }^{9}$ In cases of doubt about the text or the diagnosis (code) consensus was reached in discussion with HJB.

The participating GPs use a problem-oriented registration method, in which a CEC is defined by the GP as: any EC which needs continuous medical attention or monitoring and/or has lasted, or is likely to last, for more than 6 months and/or any recurrent EC (presented more than four times per half year). ${ }^{10}$

We registered whether a RFEs was linked to a CEC (linked to a list of all ongoing chronic or repetitive medical problems, the problem list) or to a TEC (see figure 2).

\section{Outcome measures}

Outcome measures were the number of RFEs linked to TECs for non-FAs and FAs of increasing duration in 2009 and the change in the mean number of TECs associated with the duration of FA-ship.

\section{Statistical analysis}

Patients were clustered within five GPs who worked in the centre. We calculated descriptive statistics to describe the sample, such as the number of patients and consultations, the number of presented RFEs linked to CECs and TECs and the ratio of TECs/CECs of all presented ECs for FAs of different duration. To estimate the effect of the different durations of frequent attendance on the mean number of TECs, we performed, as our main analysis, a mixed linear regression analysis (xtmixed command in Stata), with the physicians as a random intercept. Age and the number of problems on the problem list were entered in the model as continuous covariates, and sex as dummy variable. Since the number of TECs was non-normally distributed, that is, the values 0 and 1 visit were over-represented, we performed two sensitivity analyses: a mixed-effects negative binomial model (menbreg command) and quantile regression on the median (qreg command with robust variance estimation). These sensitivity analyses used the same covariates as the mixed-effects linear model. Analyses were performed using Stata, V.13.1 (College Station, Texas, USA).

\section{Ethics approval}

The study was conducted according to the Dutch legislation on data protection (Ministry of Justice, the
Figure 2 The process of coding and registration of all reasons for encounter.

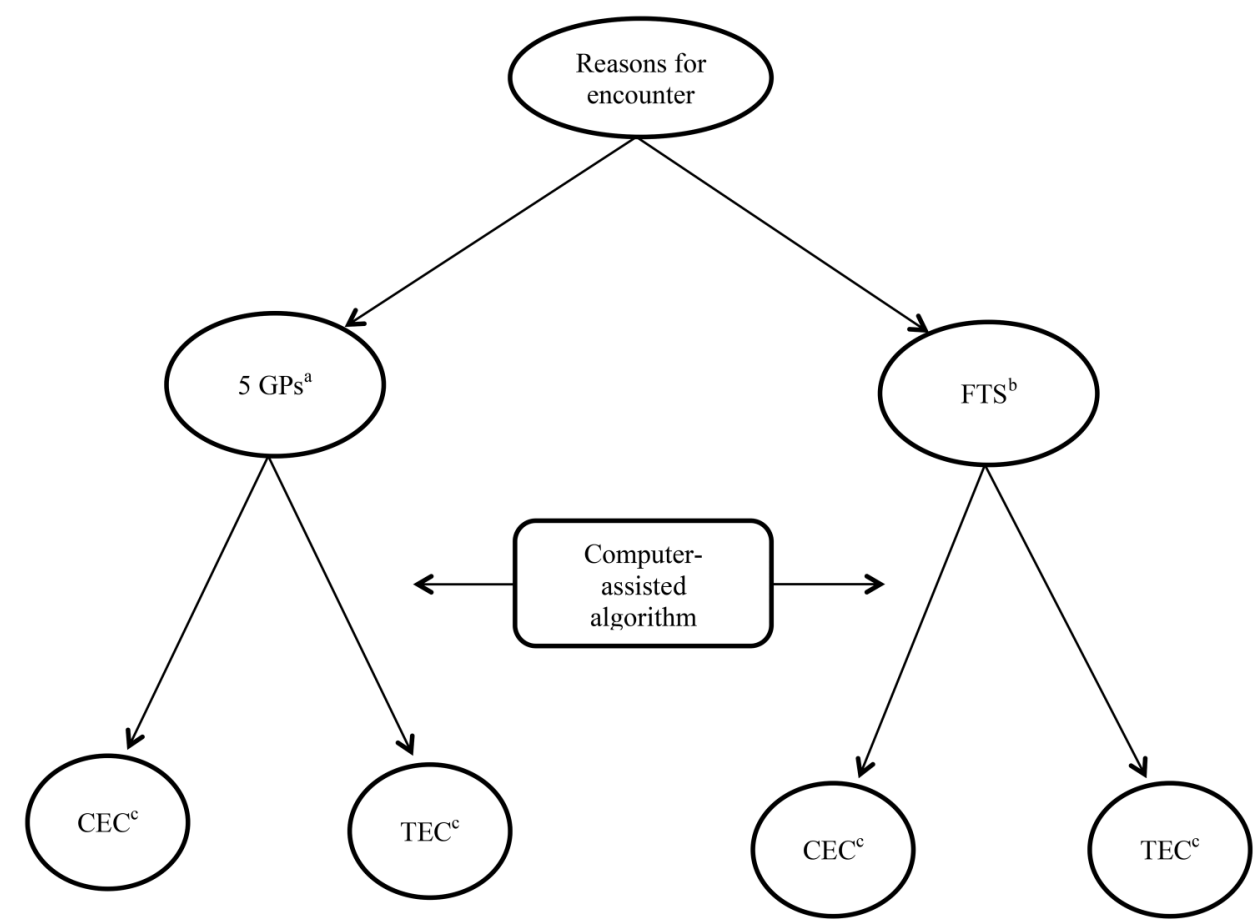

${ }^{\mathrm{a}}$ GP denotes General Practitione
${ }^{\mathrm{b}} \mathrm{FTS}$ denotes the first author

CEC denotes Chronic Episode of Care and TEC denotes Transient Episode of Care 
Netherlands). Ethics approval was waived by the Medical Ethics Committee of the Academic Medical Center of the University of Amsterdam.

\section{RESULTS}

\section{Patient population}

Out of 7500 patients of five GPs in one primary healthcare centre, 5712 patients aged 18 or older, enlisted from 2007 to 2009 provided data for this study. The numbers of included patients per GP were 1243, 1155, 1142, 1180 and 992. Of these patients 4594 were non-FA, 799 1yFA, 220 2yFA and 99 3yFA. Despite our proportional selection method (per age and sex group) FAs of longer duration were older (median age of non-FAs and 3yFAs, 45.14 (IQR 31-57) and 53.19 (IQR 42-61) years, respectively) and more often women (53.5\% and $60.6 \%$, respectively). The mean consultation frequencies in 2009 for non-FAs, 1yFAs, 2yFAs and 3yFAs were 1.81 (IQR 0-3), 4.98 (IQR 2-7), 7.68 (IQR 5-10) and 10.58 (IQR 7-12), respectively. See figure 3 and table 1 for more details.

\section{Morbidity}

In 2009, the 5712 patients presented 18767 RFEs in 15116 face-to-face consultations, of which 7923 were linked to CECs and 10864 to TECs. Per patient both the average number of RFEs, linked to TECs (non-FAs 1.4; 1yFAs 3.3; 2yFAs 5.2 and 3yFAs 7.3), and the average number of RFEs, linked to CECs, increased substantially (non-FAs 0.9; 1yFAs 2.8; 2yFAs 4.9 and 3yFAs 6.2) with the duration of frequent attendance. The ratio of RFEs linked to TECs to all ECs differed little between non-FAs $(61.4 \%)$ and all groups of FAs (52.0-54.1\%; see table 1).

The mixed linear regression analysis showed a robust effect of the duration of frequent attendance on the number of TECs, both crude and adjusted. Compared with non-FAs, the adjusted additional number of TECs for 1yFAs, 2yFAs and 3yFAs was 3.4 (95\% CI 3.2 to 3.7), 6.6 (95\% CI 6.1 to 7.0 ) and 9.4 (95\% CI 8.8 to 10.1 ), respectively (see table 2 ). The two sensitivity analyses (a mixed-effects negative binomial model (A) and a quantile regression on the median (B)) showed a similar effect of the duration of frequent attendance on TECs (see online supplementary additional file $1 \mathrm{~A}$ and $1 \mathrm{~B}$ ).

\section{DISCUSSION}

We found that, in consultations with FAs, GPs register more RFEs linked to TECs than to CECs. Both the number of RFEs linked to TECs and CECs, per patient increased with the duration of frequent attendance. The ratio of TECs to all ECs differed little for FAs and non-FAs. A multilevel regression analysis showed a substantial independent effect of frequent attendance of longer duration on the number of TECs. The constant ratio of TECs might be a sign of a low threshold for FAs to consult their GP. The large numbers of TECs in FAs might be associated with their high level of anxiety and low mastery. Therefore, the consultation pattern of FAs may best be characterised by describing both TECs and CECs.

\section{Strengths and limitations of this study}

Strength of our study is the longitudinal character of the data set. The involved GPs are stimulated to improve the registration of CECs (the problem list) by automated computer-assisted algorithms and regular feedback on their registration activity. ${ }^{10}$ By using this automated coding system coding differences between GPs are expected to be small. The GPs were not blinded for the FA status. But these frequent contacts actually helped the GPs to better distinguish between chronic and transient problems and avoided misclassifications. Although

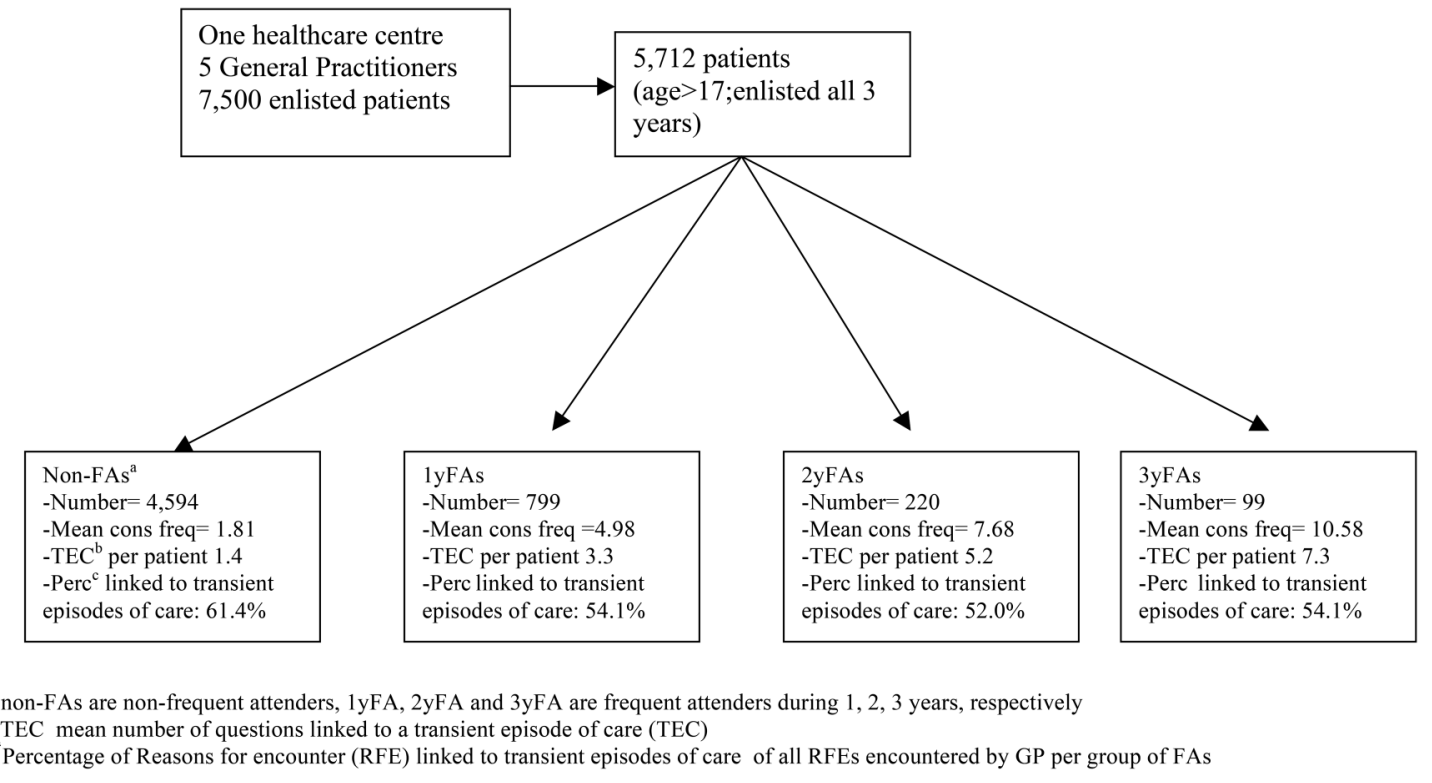

Figure 3 Selected patients and the different groups of frequent attenders. GP, general practitioner. 


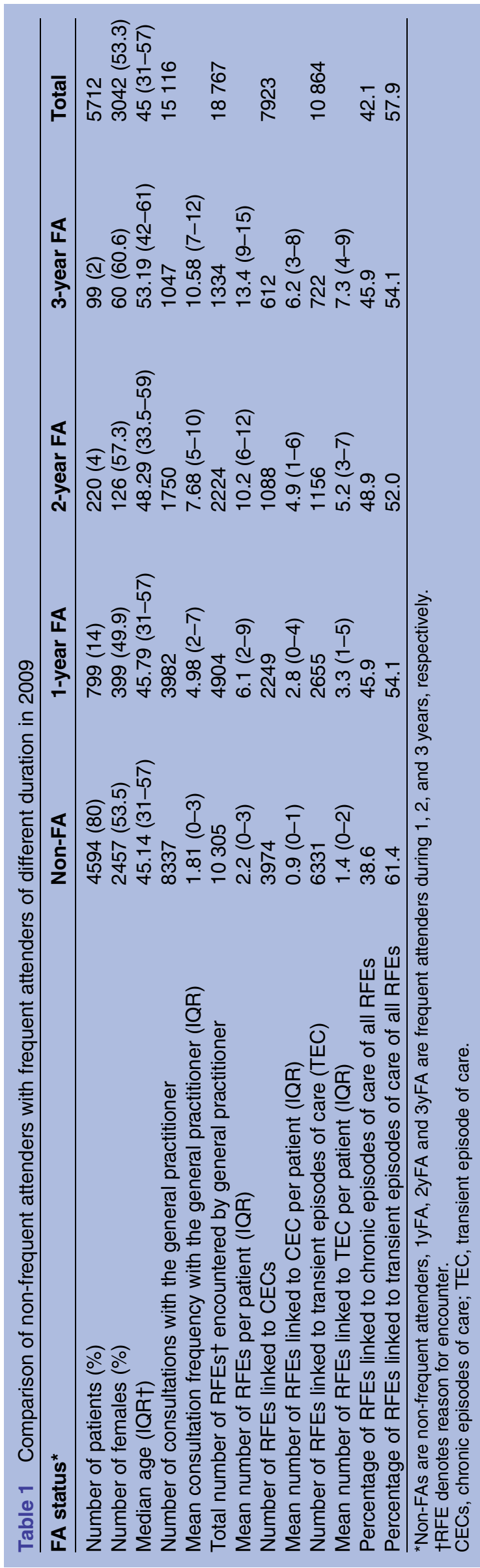

one would expect more RFEs linked to CECs in (persistent) FAs (frequent consultations and more problems on the problem lists), we found more TECs in FAs of longer duration, also after adjustment for age, sex and the number of problems. Our retrospective study was based on routinely and prospectively collected data reflecting every day, urban general practice in the Netherlands.

We also see limitations: patients often present several RFEs during a consultation and there may be differences in (under) registering and coding of ECs by GPs. Since in 2009 the involved GPs agreed to register and code CECs (linked to the problem list) only, under-registration of RFEs, linked to TECs, may be likely that year. This may have led to underestimation of the number of TECs. Categorising of RFEs as TECs/CECs may be difficult particularly in patients with somatoform disorders. Since GPs might not have recognised RFEs as part of a (somatoform) CEC, this may have led to a small underestimation of CECs. However, the random effect of the GP factor was small $(0.24 ; 95 \%$ CI 0.12 to 0.48$)$ and separate linear regression analyses per GP showed stable effects across GPs (data not shown). The five GPs linked many RFEs to CECs, but many other RFEs were left unanalysed by the GPs. FTS retrospectively analysed the text of these RFEs and coded them using the computer-assisted algorithm. Of these, very few were linked to a CEC. All other RFEs mostly pertained to ECs with low medical significance, for example, an upper respiratory infection, and were incompatible with our definition of a CEC. These RFEs were registered as a TEC. The main assignment of RFEs as CEC or TEC proved to be not difficult. The planned consensus with the second author $(\mathrm{HJB})$ was not necessary.

Some authors have suggested that consultations with other practice staff (practice assistant, practice nurse) might replace GP-patient contacts, in particular CECs, resulting in bias in FA research (off-utilization bias). ${ }^{11}$ However, the number of staff consultations in 2009 was limited and ECs presented to staff usually involved urinary tract (tests; TECs) and (monitoring of) diabetes mellitus (CECs; $36 \%$ and 25\%, respectively). Since we calculated only GP-patient contacts to determine the FA status and most staff consultations are CEC-related, we do not think these staff consultations biased our results (see online supplementary additional file 2 ).

This study originates in one urban healthcare centre with GPs involved in medical education and research in the specific Dutch healthcare system. A GP in an academic GP practice might register more CECs and fewer TECs. But this does not affect our results qualitatively, because any confounding would have worked in the direction of null findings. This may, however, restrict the generalisability of our results to similar circumstances in countries with a similar healthcare system. ${ }^{1} 1213$

Finally, because of the retrospective character of this study, we have no data on anxiety and mastery in this study. 
Table 2 The effect of frequent attender status on the number of TECs ${ }^{*}$

\begin{tabular}{|c|c|c|c|c|}
\hline & $\begin{array}{l}\text { Additional mean } \mathrm{N} † \\
\text { of TECs } ¥ \text { (crude) }\end{array}$ & $95 \% \mathrm{Cl}$ & $\begin{array}{l}\text { Additional mean N } \\
\text { of TECs (adjusted) }\end{array}$ & $95 \% \mathrm{Cl}$ \\
\hline Frequent attenders during 1 year & 3.90 & 3.65 to 4.16 & 3.41 & 3.18 to 3.65 \\
\hline Frequent attenders during 2 years & 7.62 & 7.16 to 8.07 & 6.55 & 6.13 to 6.97 \\
\hline Frequent attenders during 3 years & 11.23 & 10.56 to 11.90 & 9.43 & 8.81 to 10.05 \\
\hline Female sex§ & & & 1.38 & 1.22 to 1.54 \\
\hline Age in 2009 & & & 0.02 & 0.02 to 0.03 \\
\hline Number of problems & & & 0.50 & 0.45 to 0.55 \\
\hline Random effect GPף, SD & & & 0.24 & 0.12 to 0.48 \\
\hline Residual effect, SD & & & 3.03 & 3.00 to 3.08 \\
\hline \multicolumn{5}{|c|}{$\begin{array}{l}\text { "Results based on a multilevel linear regression analysis with two levels (GPs and patients) and non-frequent attenders as the reference } \\
\text { group. Crude and adjusted for sex, age and the number of problems on the problem list. } \\
\text { †N denotes number. } \\
\text { †TEC denotes transient episodes of care. } \\
\text { §Reference: male sex. } \\
\text { ๆGP denotes general practitioner. }\end{array}$} \\
\hline
\end{tabular}

\section{Comparison with existing literature}

Evidence about the transition from reason for encounter to TECs/CECs shows that both RFE and EC data are appropriate tools to study the process of diagnosis in general practice. ${ }^{14}{ }^{15}$ In our data, we did not code the RFE, but only the preliminary conclusion or diagnosis by the GP (EC) and whether the RFE was linked to a TEC or CEC (linked to the problem list). We were unable to find any study distinguishing between TEC and CEC diagnoses in FAs. Many articles about FAs are unclear about whether they differentiate between problem, EC or RFE diagnoses. ${ }^{2} 3$

\section{Implications for future research or clinical practice}

The many RFEs linked to a TEC in FAs may be interpreted as a result of higher levels of discomfort, (health) anxiety, hypochondriac thoughts, negative life events and low mastery. ${ }^{6}{ }^{7}$ In clinical practice, we suggest that GPs more often try to interpret RFEs linked to TECs, examine these patients for the presence of underlying anxiety disorders and/or social problems, and try to group TECs more often in a CEC (eg, anxiety disorder, social problem, personality disorder). Future research must clarify the impact of TECs on (persistence of) frequent attendance, on the costs of healthcare of FAs and explore possible ways to better manage these ECs in FAs. Awareness that persistence of frequent attendance may be aggravated by anxiety, illness behaviour and low mastery; paying more attention to these aspects in consultations with FAs may foster a more sustained, targeted intervention, instead of reacting unsystematically to new symptoms repeatedly. ${ }^{6} 16$

\section{CONCLUSION}

We found that FAs present both more CECs and more TECs with longer duration of frequent attendance. We saw no trend in the ratio of questions linked to TECs across the three groups of FAs. Since the adjusted effect of frequent attendance on the number of TECs was robust, the consultation pattern of FAs is perhaps best characterised by describing both TECs and CECs. The constant ratio of TECs might be a sign of low threshold for FAs to consult their GP. It is tempting to interpret the large number of TECs in FAs as a sign of anxiety and of low mastery. We advocate analysing all ECs in future FA research.

Acknowledgements The authors thank the PCPs of Healthcare centre Reigersbos, Amsterdam, the Netherlands for their continuous efforts to keep the electronic medical records updated and for their support to them.

Contributors FTS drafted and designed the article, monitored the data collection, coded the missing EC codes, analysed the data, and drafted and revised the paper. He is the guarantor. GtR commented, revised and supervised the paper and did the statistical analyses. HJB monitored the patient data collection, analysed data and drafted, read and revised the paper. AHS, GtR and HCPMvW supervised the project, read and revised the paper. All authors read and approved the final version of this paper.

Funding This work was supported by the Netherlands Organisation for Health Research and Development (ZonMw; Alledaagse ziekten) grant number 42011002.

Competing interests FTS worked as a GP in Health Centre Reigersbos, Amsterdam, the Netherlands from 1982 to April 2016. Between 2009 and 2014 he was a PhD at the department of General Practice of the University of Amsterdam, the Netherlands on (persistent) Frequent Attenders (PERFACTIO project).

Provenance and peer review Not commissioned; externally peer reviewed.

Data sharing statement Additional data can be accessed via the Dryad data repository at http://datadryad.org/ with the doi:10.5061/dryad.0s0n6.

Open Access This is an Open Access article distributed in accordance with the Creative Commons Attribution Non Commercial (CC BY-NC 4.0) license, which permits others to distribute, remix, adapt, build upon this work noncommercially, and license their derivative works on different terms, provided the original work is properly cited and the use is non-commercial. See: http:// creativecommons.org/licenses/by-nc/4.0/

\section{REFERENCES}

1. Smits FT, Brouwer HJ, ter Riet G, et al. Epidemiology of frequent attenders: a 3-year historic cohort study comparing attendance, morbidity and prescriptions of one-year and persistent frequent attenders. BMC Public Health 2009;9:36.

2. Gill D, Sharpe M. Frequent consulters in general practice: a systematic review of studies of prevalence, associations and outcome. J Psychosom Res 1999;47:115-30. 
3. Vedsted P, Christensen MB. Frequent attenders in general practice care: a literature review with special reference to methodological considerations. Public Health 2005;119:118-37.

4. Pymont $\mathrm{C}$, Butterworth $\mathrm{P}$. Longitudinal cohort study describing persistent frequent attenders in Australian primary healthcare. BMJ Open 2015;5:e008975.

5. Patel S, Kai J, Atha C, et al. Clinical characteristics of persistent frequent attenders in primary care: case control study. Fam Pract 2015;32:624-30.

6. Smits FT, Brouwer $\mathrm{HJ}$, Zwinderman $\mathrm{AH}$, et al. Why do they keep coming back? Psychosocial etiology of persistence of frequent attendance in primary care: A prospective cohort study.

J Psychosom Res 2014;2014:10.

7. Rosendal M, Carlsen AH, Rask MT. Symptoms as the main problem a cross-sectional study of patient experience in primary care. BMC Fam Pract 2016;17:29.

8. Smits FT, Mohrs JJ, Beem EE, et al. Defining frequent attendance in general practice. BMC Fam Pract 2008;9:21.

9. Lamberts $\mathrm{H}$, Wood M. International classification of primary care. Oxford: Oxford University Press, 1988.

10. Brouwer HJ, Bindels PJ, van Weert HC. Data quality improvement in general practice. Fam Pract 2006;23:529-36.
11. Bellón JA, Delgado-Sánchez A, de Dios Luna J, et al. Patient psychosocial factors and primary care consultation: a cohort study. Fam Pract 2007;24:562-9.

12. Smits FT, Brouwer $\mathrm{HJ}$, Zwinderman $\mathrm{AH}$, et al. Morbidity and doctor characteristics only partly explain the substantial healthcare expenditures of frequent attenders: a record linkage study between patient data and reimbursements data. BMC Fam Pract 2013;14:138.

13. Smits FT, Brouwer HJ, van Weert HC. Predictability of persistent frequent attendance: a historic 3-year cohort study. Br J Gen Pract 2009; 59:e44-50.

14. Lamberts $\mathrm{H}$, Hofmans-Okkes I. Episode of care: a core concept in family practice. J Fam Pract 1996;42:161-7.

15. Soler JK, Okkes I, Oskam S, et al. An international comparative family medicine study of the Transition Project data from the Netherlands, Malta and Serbia. Is family medicine an international discipline? Comparing incidence and prevalence rates of reasons for encounter and diagnostic titles of episodes of care across populations. Fam Pract 2012;29:283-98.

16. Smits FT. Why do they keep coming back? Persistent frequent attenders in primary care [thesis]. http://hdl.handle.net/11245/1. 430856 The Netherlands: Department of General Practice, University of Amsterdam, 2014. 